\title{
Status of the PINGU Detector
}

\author{
The IceCube-Gen2 Collaboration ${ }^{1}$, \\ ${ }^{1}$ http://icecube.wisc.edu/collaboration/authors/icrc15_gen2 \\ E-mail: kclark@physics.utoronto.ca
}

\begin{abstract}
The unknown neutrino mass ordering is one of the most interesting open questions in particle physics today, and is one of the focuses of the neutrino physics community. The measurement of a relatively large mixing angle between the first and third neutrino states makes it possible to consider several methods to measure the ordering. One such method uses atmospheric neutrinos with a large water Cerenkov detector to make the determination. A proposed expansion of the IceCube/DeepCore detector called PINGU is made up of additional strings of optical sensors (similar to those already deployed in the IceCube detector) which will be located in the ice at the centre of IceCube. The spacing between these sensors would be smaller than those of the existing DeepCore detector (both vertically and horizontally) and this increased density would lower the neutrino detection threshold substantially below $10 \mathrm{GeV}$. The physical nature of the detector as well as the methods used to make this measurement are presented.
\end{abstract}

Corresponding authors: Kenneth Clark ${ }^{* 1}$

${ }^{1}$ Department of Physics, 60 St. George Street, Toronto, ON, Canada, M5S 1A7

The 34th International Cosmic Ray Conference,

30 July- 6 August, 2015

The Hague, The Netherlands

${ }^{*}$ Speaker. 


\section{Introduction}

Neutrino oscillations have been detected in a wide variety of sources such as reactors, the atmosphere and the Sun [1]. The combination of the results from these diverse experiments presents a consistent model for neutrino oscillations. This is summarized by the PMNS matrix [2] in which the entries represent the probability that each mass eigenstate will be detected as a particular flavour eigenstate. Currently the differences in mass between the eigenstates is known, but the ordering is not. The two possibilities are the case in which the third mass eigenstate $\left(v_{3}\right)$ is the heaviest (the "normal" hierarchy) or that in which it is lightest (the "inverted" hierarchy). The distinction between these two situations is discernible using atmospheric neutrinos using an appropriate detector $[3,4]$.

The proposed PINGU detector adds optical sensors to the existing IceCube/DeepCore array in the South Pole ice, thereby leveraging the success of the established infrastructure. The IceCube detector consists of 86 strings each with 60 optical sensors deployed between depths of 1450 and $2450 \mathrm{~m}$ below the surface of the ice cap. Here, 78 strings are installed at an average horizontal spacing of $125 \mathrm{~m}$ with sensors at an average vertical spacing of $17 \mathrm{~m}$. These 4680 optical sensors are augmented with an additional 8 strings deployed in the centre of the array with horizontal spacing ranging from 40 to $70 \mathrm{~m}$ and a vertical spacing of $7 \mathrm{~m}$ (called DeepCore) which lowers the energy threshold for neutrino detection from roughly 100 to $10 \mathrm{GeV}$ [5]. The PINGU detector would further lower this threshold while increasing the sample size at all energies.

\subsection{Neutrino Studies}

The accepted framework describing neutrino oscillations has been described in detail elsewhere [1] and will only be summarized here. The existence of oscillations defines a probability that a neutrino traveling in one flavour eigenstate will interact as another eigenstate. In the simplified two neutrino scenario, a neutrino with energy $E_{v}$ having traveled a distance $L$ in eigenstate $\alpha$ will interact as eigenstate $\beta$ as shown in Equation 1.1.

$$
P\left(v_{\alpha} \rightarrow v_{\beta}\right)=\sin ^{2}(2 \theta) \sin ^{2}\left(\Delta m^{2} \frac{L}{4 E_{v}}\right)
$$

It is clear from Equation 1.1 that in the two neutrino scenario, the oscillation probability depends on the ratio of the length traveled and the energy of the neutrino as well as the mixing angle between the two states. This relationship is the same in the more complex scenarios involving three (or more) flavours of neutrinos, and proves to be an ideal parameterization with which to characterize the oscillations. IceCube analyses presented here have assumed a three flavour scenario which introduces three mixing angles, two mass-squared differences and one complex phase to this equation.

\section{IceCube/DeepCore}

The IceCube/DeepCore detector has successfully investigated the neutrino oscillation parameters and has the goal of reducing the potential phase space available for these values. In particular, recent results [8] have examined the disappearance of atmospheric muon-type neutrinos as they 
travel through the Earth. A sample of approximately three years of data with IceCube/DeepCore has shown that the neutrino oscillation parameters (in particular $\Delta m_{32}^{2}$ and $\sin ^{2} \theta_{23}$ ) can be determined with an accuracy approaching that of dedicated oscillation experiments. The scale of the measured effect is shown in the left plot of Figure 1.
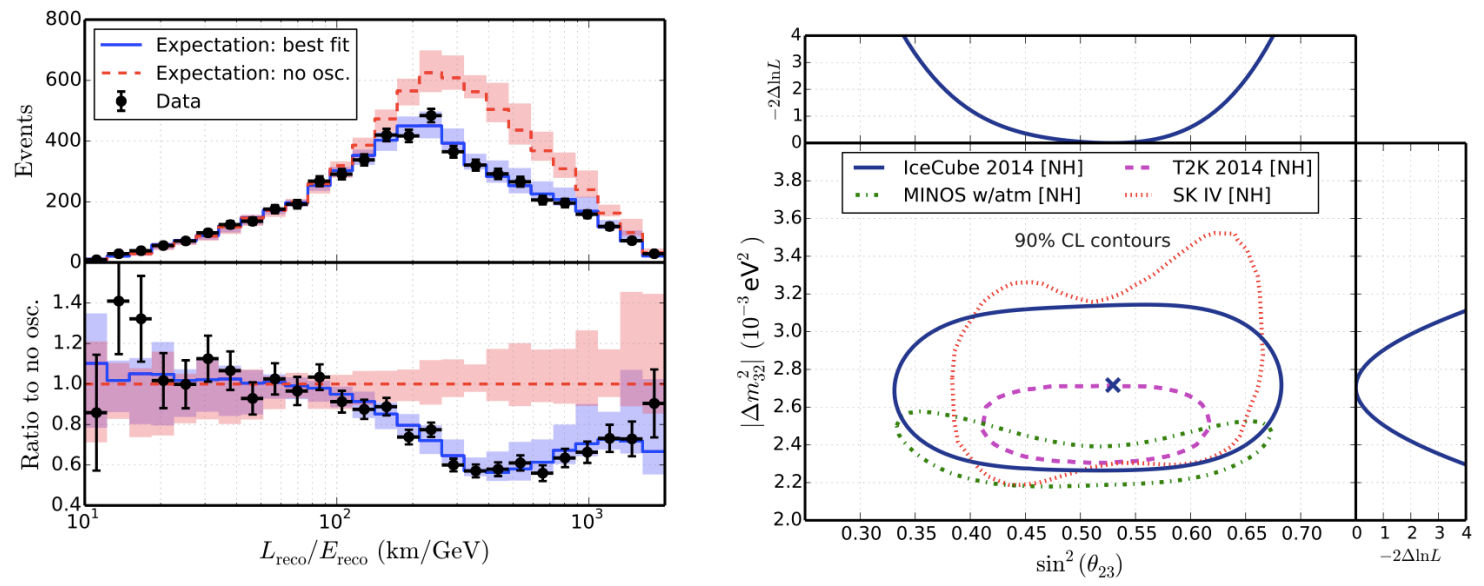

Figure 1: DeepCore analysis results from [8]. The left plot shows the reconstructed L/E distribution of events. The top portion of the left plot illustrates the best fit to the data as well as the expectation with no neutrino oscillations. The bottom portion of the left plot shows the ratio of the data and the best fit to the no-oscillation hypothesis. The bands shown are the estimated systematic uncertainties. The right plot shows the $90 \%$ confidence contours resulting from the best fit for $\sin ^{2} \theta_{23}$ and $\Delta m_{32}^{2}$ compared to results from dedicated oscillation experiments. Shown above and to the right of the plot are the likelihood profiles of the parameters. In this plot it is assumed that the normal hierarchy is true.

As an additional test of the analysis framework, the analysis shows no significant deviation from the "no oscillation model" (shown in red in the left plot of Figure 1) in the low $L / E$ region. Neutrinos in this region have a very short path length through the ice or are at very high energy, neither of which will show the effects of neutrino oscillations.

In order to determine the best fit values of the oscillation parameters, a maximum likelihood analysis is used which includes nuisance parameters accounting for the systematic uncertainties. The full three-flavour scenario for neutrino oscillations is used to determine the best fit values of $\theta_{23}$ and $\Delta m_{32}^{2}$. The $90 \%$ confidence contours for these parameters are shown in the right plot of Figure 1. As seen, the results from IceCube/DeepCore are now approaching levels of accuracy which were previously only attainable using dedicated oscillation experiments and indicate the potential of future measurements with a large-scale Cerenkov detector like PINGU.

\section{PINGU}

The PINGU detector will further lower the IceCube array energy threshold by adding another 40 strings with 96 DOMs per string. Following an extensive optimization process, the chosen geometry has a $22 \mathrm{~m}$ average horizontal spacing between strings and $3 \mathrm{~m}$ vertical spacing between modules. The new PINGU strings will be co-located with DeepCore extension at the bottom of the array in the centre of the IceCube strings, as shown in Figure 2. The effect of lowering the neutrino 
detection energy threshold is shown in the "effective area" curve for the PINGU detector in Figure 3.

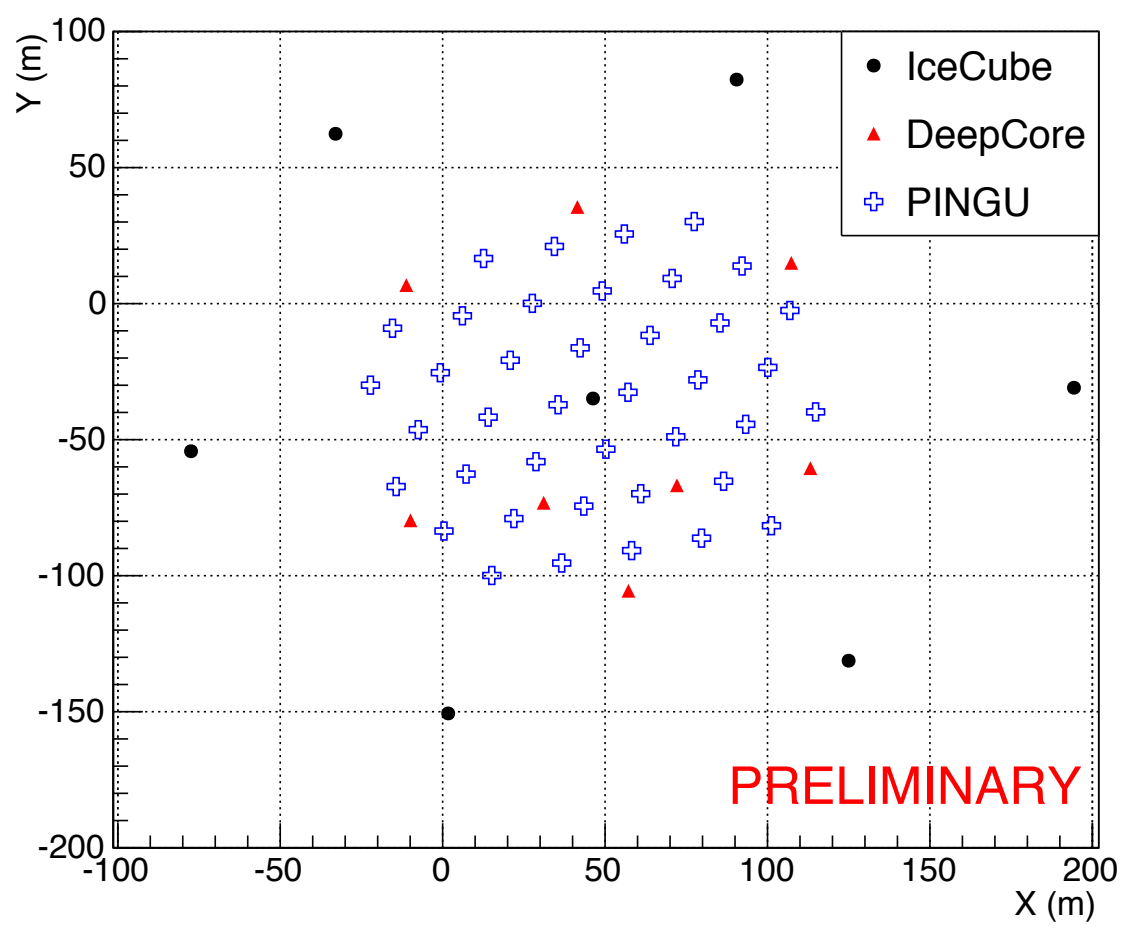

Figure 2: A top view of the optimized PINGU geometry showing the position of IceCube strings (black circles), DeepCore strings (red triangles) and planned PINGU strings (blue crosses) .

The positioning of the PINGU detector within the DeepCore fiducial volume allows the existing IceCube strings to be used to veto background muons that enter the PINGU volume. These incoming events, which produce light outside of the PINGU strings, will be removed from the signal event sample using tools which have been used successfully in the DeepCore analyses [5]. The additional PINGU DOMs also improve the reconstruction resolution by collecting more of the Cerenkov photons produced in each event. The mean resolution $\left(\left[E_{\text {true }}-E_{\text {reco }}\right] / E_{\text {true }}\right)$ in the primary region of interest for PINGU (approximately 5 to $15 \mathrm{GeV}$ ) has been decreased to roughly $30 \%$ at $5 \mathrm{GeV}$ and is better at higher energies. In addition, the angular resolution also improves with the detection of additional photons, which in turn improves the determination of the neutrino path length.

The effective area of the PINGU detector is obtained using simulated data. This calculated value represents the equivalent area in which all neutrinos incident on the Earth would be observed. Of particular note in these plots is the energy range over which the effective area extends. As shown, significant sensitivity to all neutrino flavours is maintained down to at least $5 \mathrm{GeV}$, with a sharp reduction at lower energies. It is this lowered energy limit (compared to the IceCube limit, on the order of $100 \mathrm{GeV}$, or the DeepCore limit, on the order of $10 \mathrm{GeV}$ ) which is of benefit to the planned 

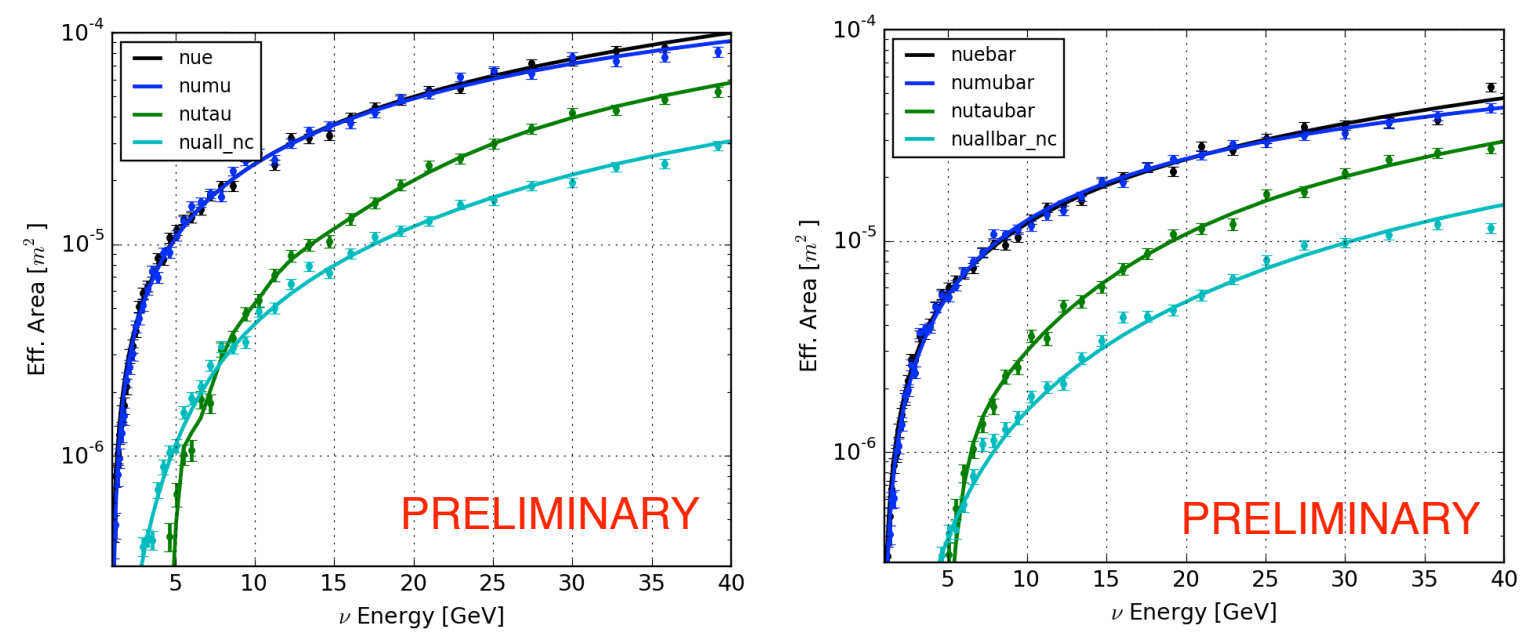

Figure 3: The effective area of the PINGU detector for all neutrino (antineutrino) flavours, left (right). In this plot, nue represents the area for electron-type neutrinos, numu represents muon-type neutrinos, nutau represents tau-type neutrinos, and nuall_nc represents neutral current neutrinos of all flavours.

future neutrino oscillation analyses.

\section{PINGU Physics}

\subsection{Neutrino Mass Ordering}

The primary goal of the PINGU detector is the determination of the neutrino mass ordering (hierarchy), an open question which has persisted for many years [1]. In the current status of the study of neutrino oscillations, many parameters are known, which aids in the determination of the ordering.

As neutrinos pass through the Earth, the flavour oscillations are described by the MSW effect [6], which results in a resonant enhancement of the probability of the $v_{\mu} \rightarrow v_{e}$ oscillation if the mass ordering is "normal" ( $v_{3}$ is heaviest) and $\bar{v}_{\mu} \rightarrow \bar{v}_{e}$ if the ordering is "inverted" ( $v_{3}$ is lightest). The increase in the oscillation probability is highly dependent on both the energy and the linear electron density along the path of the neutrino, a combination of the density profile of the Earth and the path length of the neutrino. The range of incoming zenith angles available to the PINGU detector therefore provides a wide range of oscillation probabilities.

In addition to the MSW effect, the neutrino oscillations also experience a parametric enhancement of their oscillations in specific circumstances [7]. This enhancement affects neutrinos passing through the mantle just outside of the outer core which have an energy of roughly $6 \mathrm{GeV}$. Parametric oscillation provides about one third of PINGU's sensitivity to the neutrino mass ordering.

These oscillation probability changes are dependent on the nature of the hierarchy and the neutrino $(v$ or $\bar{v})$ so that $P\left(v_{\mu} \rightarrow v_{\mu}\right)_{N H}=P\left(\bar{v}_{\mu} \rightarrow \bar{v}_{\mu}\right)_{I H}$. Since IceCube/DeepCore and PINGU are unable to distinguish between neutrinos and anti-neutrinos, the changes are visible only in the sum of these probabilities (e.g. $\left.\left[P\left(v_{\mu} \rightarrow v_{\mu}\right)_{N H}+P\left(\bar{v}_{\mu} \rightarrow \bar{v}_{\mu}\right)_{N H}\right]\right)$ which can be used to create a representative event sample for both "normal" and "inverted" hierarchy distributions. These can 
then be subtracted to show the difference in the number of events expected, and separated into "track-like" $\left(v_{\mu} C C\right)$ and "cascade-like" $\left(v_{e}, v_{\tau} C C, v_{\text {all }} N C\right)$ events using the particle identification (PID) developed for use with the reconstructions used in PINGU. The results of these plots are shown in Figure 4.
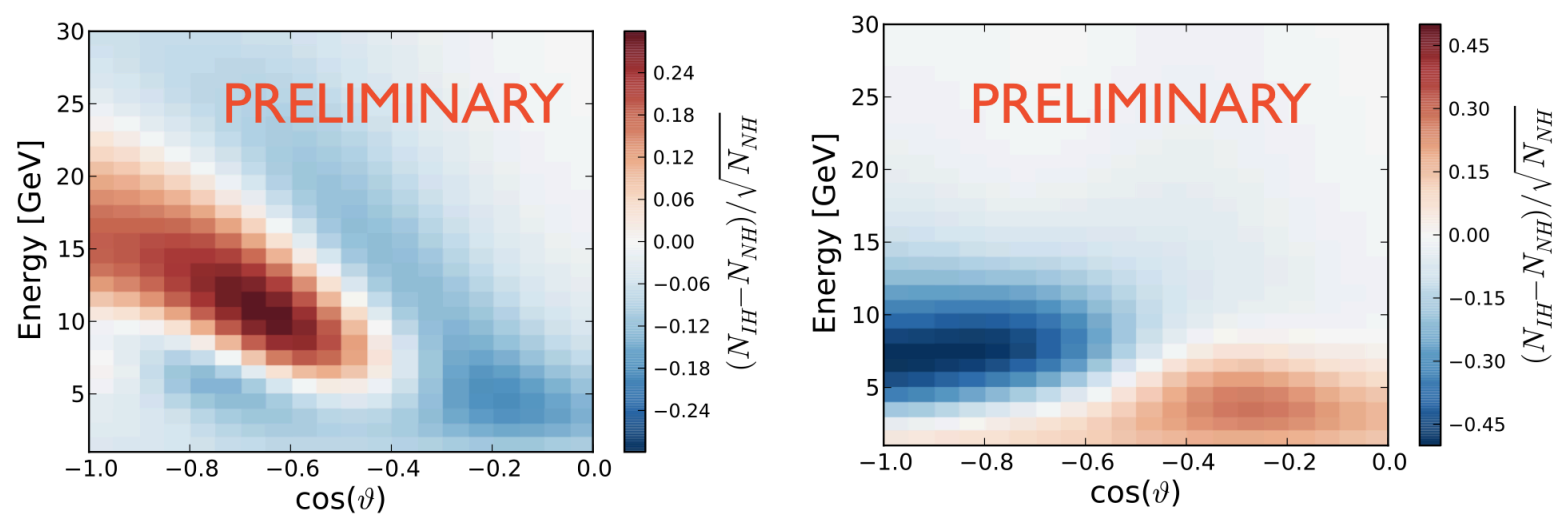

Figure 4: The scaled difference in the annual counts for the PINGU detector are shown for events identified as track-like (left) and cascade-like (right) during the reconstruction process.

The determination of the hierarchy then relies on the shape of the event distributions as shown in Figure 4. Refinements in the analysis have enhanced the sensitivity, while a more accurate noise model and a more complete set of systematic errors have partially offset this gain. The primary analysis method is now a likelihood ratio study which accounts for many of the predicted systematics, broadly separated into oscillation, flux and detector systematics. Figure 5 shows the results of extracting the neutrino mass ordering with PINGU with only statistical uncertainties (dashed lines) and with all systematics included (solid lines). As seen in Figure 5, the $3 \sigma$ threshold in this analysis is reached with just over three years of data regardless of the hierarchy, although it will be slightly sooner if the true hierarchy is normal.

\subsection{Neutrino Oscillation Parameters}

In addition to determining the true value of the neutrino mass hierarchy, the neutrino oscillation parameters (and in particular the atmospheric parameters $\theta_{23}$ and $\Delta m_{32}^{2}$ ) can also be studied using the lowered energy threshold provided by the PINGU detector. The oscillation parameters can be extracted from the likelihood-based analysis discussed in the previous section. The preliminary expectations of this analysis are shown in Figure 6. The expected improved sensitivity to lower mass WIMPs in the PINGU detector is also shown.

\subsection{Dark Matter}

In addition to studying neutrinos and their oscillations, PINGU can also search for astrophysical sources which produce neutrinos and use them as messengers. Of particular interest to the physics community is the search for dark matter. The IceCube detector and the DeepCore extension have carried out these dark matter searches, producing world-leading results as shown in the right plot of Figure 6. 


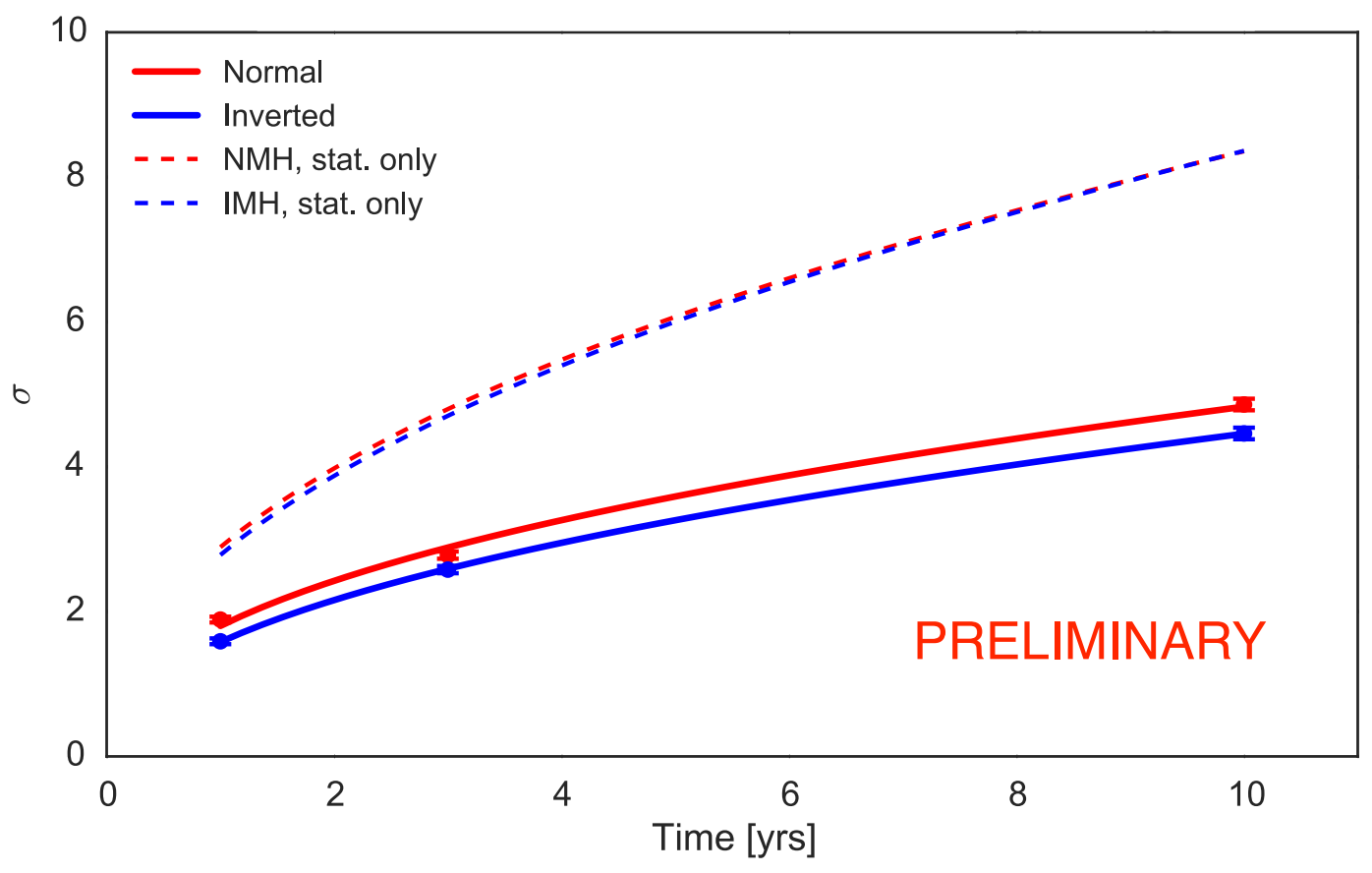

Figure 5: The evolution of the significance for determining the neutrino mass hierarchy with the PINGU detector shown by years of data taking.

The dark matter analysis has been carried out at several stages during construction of the IceCube/DeepCore array. The red curve in Figure 6 labelled IC79 represents the incomplete IceCube detector with only 79 strings, while the curve in black represents the results using the full PINGU detector (which includes the DeepCore extension). The blue bands indicate the range expected from anticipated improvements to the analysis methods.

The increased density of photodetectors in the ice will aid the results curve in two primary ways. The first is that the energy threshold of the detector will be lowered, making available more neutrinos at low energies. This lowering will change the sensitive phase space for the PINGU detector to include much lower energy events. The second way the results will be changed is that the reconstructions will be improved, as discussed previously. Improving the reconstruction for the dark matter analysis makes a significant difference, since the pointing accuracy of the detector can be used to distinguish signal from background, particularly for the analysis of solar WIMP annihilations.

\section{Summary}

The IceCube array, with its low energy extension, have shown that the execution of precision particle physics is achievable in the Antarctic ice at the South Pole. The planned PINGU sub-array holds the potential to further lower the neutrino detection energy threshold, making it possible to study the physics of the neutrino mass ordering and access low-mass dark matter. The optimization 

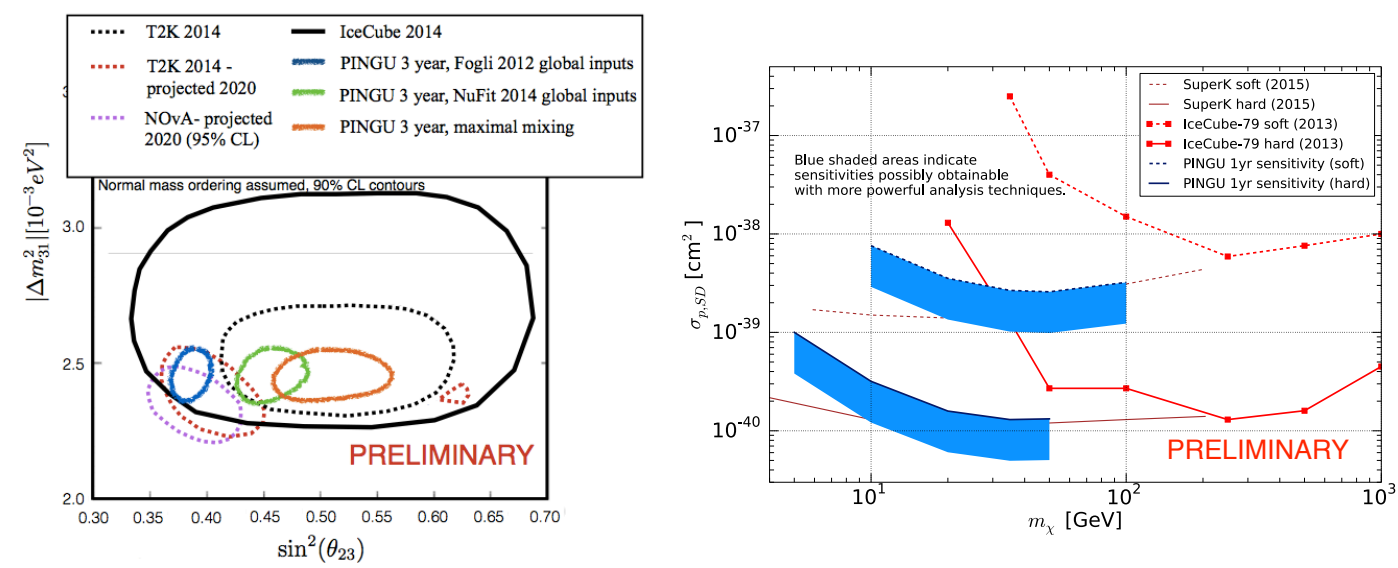

Figure 6: Additional physics results using the PINGU detector. The sensitivity to the oscillation parameters is shown in the right plot with the PINGU projected sensitivity. The left plot shows the dark matter exclusion limit results for SuperK, IceCube and the projected results for PINGU.

studies of PINGU are now nearing completion and the collaboration has started planning for the next stage of the project.

\section{References}

[1] J. Beringer et al, Review of Particle Physics, Phys. Rev. D 86 (2012) 010001

[2] Z. Maki, M. Nakagawa, and S. Sakata, Remarks on the unified model of elementary particles, Prog. Theor. Phys. 28 (1962) 870

[3] O. Mena, I. Mocioiu, and S. Razzaque, Neutrino mass hierarchy extraction using atmospheric neutrinos in ice,Phys. Rev. D 78 (2008) 093003

[4] E. Kh. Akhmedov, S. Razzaque, and A. Yu. Smirnov, Mass hierarchy, 2-3 mixing and CP-phase with huge atmospheric neutrino detectors,Journal of High Energy Physics 82 (2013)

[5] The IceCube/DeepCore Collaboration, The design and performance of IceCube DeepCore, Astroparticle Physics 35 (2012) 615

[6] L. Wolfenstein, Neutrino Oscillations in Matter, Phys. Rev. D 17 (1978) 2369; S. P. Mikheyev and A. Yu. Smirnov Resonance enhancement of oscillations in matter and solar neutrino spectroscopy, Sov. J. Nucl. Phys. 42 (1985) 913

[7] E. Kh. Akhmedov, Parametric resonance of neutrino oscillations and passage of solar and atmospheric neutrinos through the Earth, Nuclear Physics B 538 (1999) 25

[8] The IceCube/DeepCore Collaboration, Determining neutrino oscillation parameters from atmospheric muon neutrino disappearance with three years of IceCube DeepCore data, Phys. Rev. D 91 (2015) 072004 